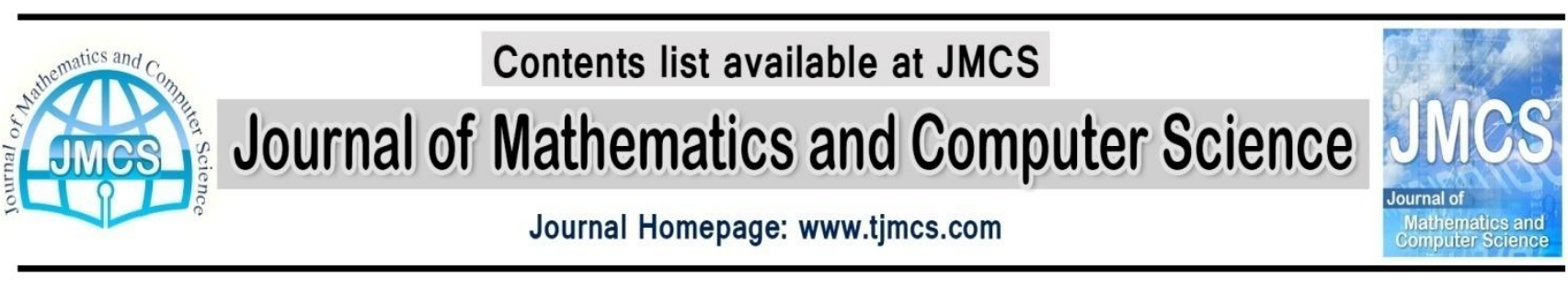

\title{
Ones Assignment Method for Solving Traveling Salesman Problem
}

\author{
Hadi Basirzadeh \\ Department of Mathematics, Shahid Chamran University, Ahvaz, Iran
}

Article history:

\author{
basirzad@scu.ac.ir
}

Received April 2014

Accepted May 2014

Available online May 2014

\begin{abstract}
This paper presents an approach namely, ones assignment method, for solving the traveling salesman problem. We have previously used this method for the assignment problem. We have slightly modified the procedure to get a tour of the traveling salesman problem.

First we define the distance matrix, then by using determinant representation we obtain a reduced matrix which has at least one 1 in each row and each column. Then by using the new method, we obtain an optimal solution for traveling salesman problem by assigning ones to each row and each column. The new method is based on creating some ones in the distance matrix and then try to find a complete solution to their ones. At the end, this method is illustrated with some numerical examples.
\end{abstract}

Keywords: Assignment problem, Linear integer programming, traveling salesman problem.

\section{Introduction}

An important topic, put forward immediately after the transportation problem, is the assignment problem. This is particularly important in the theory of decision making. The assignment problem is one of the earliest application of linear integer programming problem. Different methods have been presented for assignment problem and various articles have been published on the subject. See [4], [5] and [7] for the history of these methods. Recently, we introduce a quick method to calculate the super efficient point in multi objective assignment problems [2].

A considerable number of methods has been so far presented for assignment problem in which the Hungarian method is more convenient method among them. This iterative method is based on add or subtract a constant to every element of a row or column of the cost matrix, in a minimization model and create some zeros in the given cost matrix and then try to find a complete assignment in terms of zeros. By a complete assignment for a cost $n \times n$ matrix, we mean an assignment plan containing exactly $n$ assigned independent zeros, one in each row and one in each column. The main concept of assignment 
problem is to find the optimum allocation of a number of resources to an equal number of demand points. An assignment plan is optimal if optimizes the total cost or effectiveness of doing all the jobs. One of the problems similar to that an assignment problem is the traveling salesman problem (TSP). Historically the TSP deals with finding the shortest tour in an n-city situation where each city is visited exactly once. In this problem, traveling salesman wants to minimize the total distance traveled ( or time or money) during his visit of $\mathrm{n}$ cities. $d_{i j}, i=1,2, \ldots, n$ is the distance of $i$ th node to $j$ th node. Note that $d_{i j}=-$ when $i=j$, i.e. we do not produce the item $i$ again after $i$. The individual set up costs can be arranged in the form of a square matrix. A traveling salesman problem is to determine a set of $n$ elements of this matrix , one in each row and one in each column, so as to minimize the sum of elements determined above. Traveling salesman problem is similar to the assignment problem, but here two extra restrictions are imposed. The first restriction is that we can not select the element in the leading diagonal as we do not follows $i$ again by $i$. The second restriction is that we do not produce an item again until all the items are produced once. The second restriction means no city is visited twice until the tour of all the cities is completed. This paper attempts to propose a method, namely ones assignment method, for solving traveling salesman problem, which is different from the preceding methods.

This method is a heuristic method which we applied it for solving assignment problem, see [1].

Mathematically a traveling salesman problem can be stated as follows: Optimize

$$
\sum_{i=1}^{n} \sum_{j=1}^{n} d_{i j} x_{i j}
$$

subject to

$$
\left.\begin{array}{l}
\sum_{j=1}^{n} x_{i j}=1, \quad i=1, \ldots, n \\
\sum_{i=1}^{n} x_{i j}=1, \quad j=1, \ldots, n \\
x_{i j}=0 \text { or } 1, \quad i=1, \ldots, n, j=1, \ldots n . \\
\text { The first and } \text { sec } \text { ond restriction. }
\end{array}\right\}
$$

Where $d_{i j}$ is the distance from city $i$ to city $j$, and $x_{i j}$ is to be some positive integer or zero, and the only possible integer is one, so the condition of $x_{i j}=0$ or 1 , is automatically satisfied.

Associated to each traveling salesman problem there is a matrix called distance matrix $\left[d_{i j}\right]$ where $d_{i j}$ is the distance from city $i$ to city $j$. In this paper we call it distance matrix, and represent it as follows:

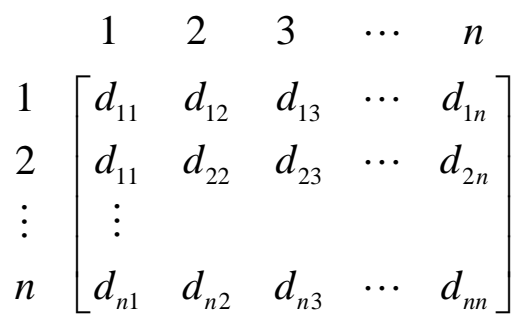

which is always a square matrix, thus each city can be assigned to only one city.

In fact any solution of this problem will contain exactly $m$ non-zero positive individual allocations. 
A customary and convenient method, termed as "assignment algorithm" has been developed for such problems. This iterative method is known as Hungarian assignment method. It is based on add or subtract a constant to every element of a row or column of the cost matrix in a minimization model, and create some zeros in the given cost matrix and then try to find a complete assignment in terms of zeros. In fact our aim is to create some ones in place of zeroes on distance matrix, and try to assign them in our problem.

\section{Our approach for solving traveling salesman problem}

This section presents a method to solve the traveling salesman problem which is different from the preceding method. We call it "ones assignment method", because of making assignment in terms of ones. The new method is based on creating some ones in the distance matrix and then try to find a complete solution in terms of ones. By a complete solution we mean an assignment plan containing exactly $\mathrm{m}$ assigned independent ones, one in each row and one in each column.

Now, consider the distance matrix where $d_{i j}$ is the distance of $i$ th city to $j$ th city.

$$
\begin{gathered}
1 \\
1 \\
2 \\
\vdots \\
n
\end{gathered}\left[\begin{array}{ccccc}
d_{11} & d_{12} & d_{13} & \cdots & d_{1 n} \\
d_{11} & d_{22} & d_{23} & \cdots & d_{2 n} \\
\vdots & & & & \\
d_{n 1} & d_{n 2} & d_{n 3} & \cdots & d_{n n}
\end{array}\right]
$$

The new algorithm is as follows:

let (1-2) be a traveling salesman problem in which the objective function can be minimized or maximized.

\section{Step 1}

In a minimization (maximization) case, find the minimum ( maximum) element of each row in the distance matrix (say $a_{i}$ ) and write it on the right hand side of the matrix.

$$
\left[\begin{array}{ccccc}
d_{11} & d_{12} & d_{13} & \cdots & d_{1 n} \\
d_{11} & d_{22} & d_{23} & \cdots & d_{2 n} \\
\vdots & & & & \\
d_{n 1} & d_{n 2} & d_{n 3} & \cdots & d_{n n}
\end{array}\right] \begin{gathered}
a_{1} \\
a_{2} \\
\vdots \\
a_{n}
\end{gathered}
$$

Then divide each element of $i$ th row of the matrix by $a_{i}$. These operations create at least one ones in each rows.

$$
\left[\begin{array}{ccccc}
d_{11} / a_{1} & d_{12} / a_{1} & d_{13} / a_{1} & \cdots & d_{1 n} / a_{1} \\
d_{11} / a_{2} & d_{22} / a_{2} & d_{23} / a_{2} & \cdots & d_{2 n} / a_{2} \\
\vdots & & & & \\
d_{n 1} / a_{n} & d_{n 2} / a_{n} & d_{n 3} / a_{n} & \cdots & d_{n n} / a_{n}
\end{array}\right] \begin{gathered}
a_{1} \\
a_{2} \\
\vdots \\
a_{n}
\end{gathered}
$$

In term of ones for each row and column do assignment, otherwise go to step 2 . 


\section{Step 2}

Find the minimum ( maximum) element of each column in distance matrix ( say $b_{j}$ ), and write it below $j$ th column. Then divide each element of $j$ th column of the matrix by $b_{j}$.

These operations create at least a one in each column. Make assignment in terms of ones. If no feasible assignment can be achieved from step (1) and (2) then go to step 3.

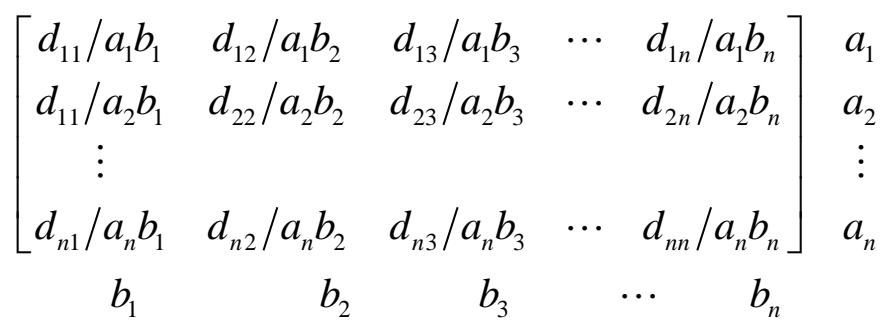

Note: In a maximization case, the end of step 2 we have a fuzzy matrix, which all elements are belong to $[0,1]$, and the greatest element is one [6].

\section{Step 3}

Draw the minimum number of lines to cover all the ones of the matrix. If the number of drowned lines less than $n$, then the complete solution is not possible, while if the number of lines is exactly equal to $n$, then the complete solution is obtained.

\section{Step 4}

If a complete solution is not possible in step 3, then select the smallest (largest) element (say $d_{i j}$ ) out of those which do not lie on any of the lines in the above matrix. Then divide by $d_{i j}$ each element of the uncovered rows or columns, which $d_{i j}$ lies on it. This operation create some new ones to this row or column.

If still a complete optimal solution is not achieved in this new matrix, then use step 4 and 3 iteratively. By repeating the same procedure the optimal solution will be obtained.

Priority plays an important role in this method, when we want to assign the ones.

\section{Priority rule}

For maximization (minimization) traveling salesman problem, assign the ones on the rows which have greatest (smallest) element on the right hand side, respectively. If a tour is not reached, so do the assignment that will make a tour.

We note that if a tour does not occur, then assign the element immediately greater than one.

One question arise here, what to do with non square matrix? To make square, a non square matrix, we add one artificial row or column which all elements are one. Thus we solve the problem with the new matrix, by using the new method. The matrix after performing the steps reduces to a matrix which has ones in each row and each column. So, the optimal solution has been reached.

\section{Mathematical concept of the subject}


One of the operations associated with matrices is calculation of scalar value known as the determinant of a square matrix [8]. Here we do not want calculate the determinant of a matrix, only we want to use the properties of the determinant operator, when we use customary and common notation, for the determinant of the matrix.

Here we use the notation $|A|$, for representation of the determinant of matrix $A$. Several basic properties of determinant are useful but one of them useful for this method. This is factorization.

\section{Proposition 1:}

When $\lambda$ (a non zero scalar) is a factor of a row (column) of $|A|$, then it is also a factor of $|A|$. That is

$$
|A|=\lambda \cdot\left|A_{\lambda}\right|
$$

Which $A_{\lambda}$ is the matrix A with $\lambda$ factored out of a row or a column of $\mathrm{A}$.

If $\mathrm{A}$ is an $n \times n$ matrix and $\lambda_{i}, i=1,2, \ldots, n$ is a factor of $i$ th row of $|A|$, then $\lambda=\lambda_{1} \cdot \lambda_{2} \ldots . \lambda_{n}$ is also a factor of $|A|$. That is

$$
|A|=\lambda \cdot\left|A_{\lambda}\right|=\lambda_{1} \cdot \lambda_{2} \ldots . \lambda_{n} \cdot\left|A_{\lambda_{1} \cdot \lambda_{2} \ldots \lambda_{n}}\right|
$$

Similarly, if $\mathrm{A}$ is an $n \times n$ matrix and $\mu_{j}, j=1,2, \ldots, n$ is a factor of $j$ th column of $|A|$, then $\mu=\mu_{1} \cdot \mu_{2} \ldots . \mu_{n}$ is also a factor of $|A|$.

$$
|A|=\mu .\left|A_{\mu}\right|=\mu_{1} \cdot \mu_{2} \ldots \mu_{n}\left|A_{\mu_{1}, \mu_{2} \ldots \mu_{n}}\right|
$$

which $A_{\mu_{1}, \mu_{2} \ldots \mu_{n}}$ is the matrix A with $\mu_{j}$ factored out of $j$ th column, $\mathrm{j}=1,2, \ldots, \mathrm{n}$.

\section{Proposition 2:}

If $\mathrm{A}$ is an $n \times n$ matrix and $\lambda$ is a factor of rows of $|A|$, and $\mu$ is a factor of columns of $|A|$, then $\lambda$. $\mu$ is also a factor of $|A|$. That is,

$$
|A|=\lambda \cdot \mu\left|A_{\lambda, \mu}\right|
$$

which $A_{\lambda \mu}$ is the matrix A with $\lambda$ factored out of a row and $\mu$ factored out of a column of $\mathrm{A}$.

Note : In fact, when we apply the ones assignment method to solve an assignment problem, we use the above propositions to reduce the matrix of the problem into a matrix which has enough ones in each rows and each columns to assign them. Here we emphasis that the locations of the ones are important to assign, and when we want to calculate the objective function of the problem, we use the real values of the assigned elements in initial distance matrix.

\section{Numerical examples}

The following examples may be helpful to clarify the proposed method:

Example 1: Consider the following traveling salesman problem. Design a tour to five cities to the salesman such that minimize the total distance. Distance between cities is shown in the following matrix. 


$$
\begin{gathered}
1 \\
1 \\
2 \\
3 \\
4 \\
5
\end{gathered}\left[\begin{array}{ccccc}
- & 10 & 3 & 6 & 9 \\
5 & - & 5 & 4 & 2 \\
4 & 9 & - & 7 & 8 \\
7 & 1 & 3 & - & 4 \\
3 & 2 & 6 & 5 & -
\end{array}\right]
$$

Find the minimum element of each row in the distance matrix (say $a_{i}$ ) and write it on the right hand side of the matrix, as follows:

1
1
2
2
3
4
5 $\left[\begin{array}{cccccc}- & 10 & 3 & 4 & 5 \\ 5 & - & 5 & 4 & 2 \\ 4 & 9 & - & 7 & 8 \\ 7 & 1 & 3 & - & 4 \\ 3 & 2 & 6 & 5 & -\end{array}\right] \begin{aligned} & 3 \\ & 2 \\ & 1 \\ & 2\end{aligned}$

Then divide each element of $i$ th row of the matrix by $a_{i}$. These operations create some ones to each row, and the matrix reduces to following matrix.

$$
\left[\begin{array}{ccccc}
- & 3.3 & 1 & 2 & 3 \\
2.5 & - & 2.5 & 2 & 1 \\
1 & 2.25 & - & 1.75 & 2 \\
7 & 1 & 3 & - & 4 \\
1.5 & 1 & 3 & 2.5 & -
\end{array}\right] \begin{aligned}
& 3 \\
& 2 \\
& 2
\end{aligned}
$$

Now find the minimum element of each column in distance matrix ( say $b_{j}$ ), and divide each element of $j$ th column of the matrix by $b_{j}$.

This operation create some ones to each row and each column.

$$
\left[\begin{array}{ccccc}
- & 3.3 & 1 & 1.14 & 3 \\
2.5 & - & 2.5 & 1.14 & 1 \\
1 & 2.25 & - & 1 & 2 \\
7 & 1 & 3 & - & 4 \\
1.5 & 1 & 3 & 1.428 & -
\end{array}\right] \begin{aligned}
& 3 \\
& 2 \\
& 2
\end{aligned}
$$

The minimum number of lines required to pass through all ones is 4 , and the minimum element of the uncovered is 1.428 on 5th row, so divide each element of 5th row of the matrix by 1.428 . 


$$
\left[\begin{array}{ccccc}
- & 3.3 & 1 & 1.14 & 3 \\
2.5 & - & 2.5 & 1.14 & 1 \\
1 & 2.25 & - & 1 & 2 \\
7 & 1 & 3 & - & 4 \\
1.05 & 0.7 & 2.1 & 1 & -
\end{array}\right] \begin{aligned}
& 3 \\
& 2
\end{aligned}
$$

Now, minimum number of lines required to pass through all the ones of the matrix is 5 . So, the complete solution is possible, and we can assign the ones, it is based on priority rule. Priority rule is assigning one on the rows which have the smallest element on the right hand side, respectively.

The details of this program are as follows:

City 1 assigns to City 3 distance 3

City 2 assigns to City 5 distance 2

City 3 assigns to City $4 \quad$ distance 7

City 4 assigns to City 2 distance 1

City 5 assigns to City $1 \quad$ distance 3

so the optimal assignment has been reached, and the optimal path is $(1,3),(3,4),(4,2),(2,5),(5,1)$ and total distance according to this plan is 16 .

Example 2: Consider the following traveling salesman problem. Design a tour to five cities to the salesman such that minimize the total distance. Distance between cities is shown in the following matrix.

1
2
3
4
5 $\left[\begin{array}{cccccc}- & 11 & 10 & 12 & 4 \\ 2 & - & 6 & 3 & 5 \\ 3 & 12 & - & 14 & 6 \\ 6 & 14 & 4 & - & 7 \\ 7 & 9 & 8 & 12 & -\end{array}\right]$

Find the minimum element of each row in the distance matrix (say $a_{i}$ ) and divide each element of $i$ th row of the matrix by $a_{i}$. These operations create ones to each rows, and the matrix reduces to following matrix.

$$
\left[\begin{array}{ccccc}
- & 2.75 & 2.5 & 3 & 1 \\
1 & - & 3 & 1.5 & 2.5 \\
1 & 4 & - & 3.5 & 2 \\
1.5 & 3.5 & 1 & - & 1.75 \\
1 & 1.28 & 1.14 & 1.71 & -
\end{array}\right] \begin{gathered}
4 \\
3 \\
7
\end{gathered}
$$

Now the minimum element of second column is 1.28 . Divide each element of second column by 1.28 and the minimum element of 4 th column is 1.5 . Divide each element of 4 th column by 1.5 .

These operations create some ones on second and 4 th column, and the reduced matrix is as follows: 


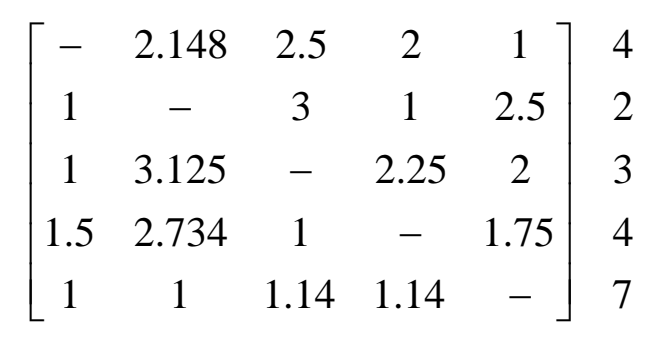

The minimum number of lines required to pass through all the ones of the matrix is 5 .

So, the complete solution is possible, and we can assign the ones, it is based on priority rule. Priority rule is assigning one on the rows which have the smallest element on the right hand side, respectively.

The details of this program are as follows:

City 1 assigns to City 5 distance 4

City 2 assigns to City $4 \quad$ distance 3

City 3 assigns to City $1 \quad$ distance 3

City 4 assigns to City $3 \quad$ distance 4

City 5 assigns to City 2 distance 9.

So the optimal path has been reached, $2-4-3-1-5-2$, and total distance according to this plan is 23 .

\section{References:}

[1] Hadi Basirzadeh, Ones assignment method for solving assignment problems, Applied Mathematical Sciences, vol 6, no. 47, 2347-2355 (2012).

[2] Hadi Basirzadeh, Vahid Morovati, Aabbas.Sayadi, A quick method to calculate the super-efficient point in multi-objective assignment problems, TJMCS, vol 10, no. 3, 157-234 (2014) .

[3] M. S. Bazarra, John J. Jarvis, Hanif D. Sherali, Linear programming and network flows, (2005).

[4] B. s. Goel, S. K. Mittal, Operations Research, Fifth Ed, (1982) 2405-2416.

[5] Hamdy A. Taha, Operations Research, an introduction, 8th Ed. (2007).

[6] H. J. Zimmermann, Fuzzy set theory and its Applications, third Ed., Kluwer Academic, Boston, 1996.

[7] AnshumanSahu, RudrajitTapador, Solving the assignment problem using genetic algorithm and simulated annealing, IJAM, (2007).

[8] Shayle R. Searle, Matrix algebra useful for statistics, John Wiley, (2006). 